\title{
A sample-to-disk system for psychomusical research
}

\author{
ROGER A. KENDALL \\ University of California, Los Angeles, Los Angeles, California
}

\begin{abstract}
A hardware-and-software system is described that permits musical-bandwidth signal quantization, monophonic or stereophonic, direct to/from hard disk. The system, which uses an IBM AT or compatible, is cost effective and device independent. Software functions allow for automated aural and graphical signal analyses. Perceptual experiments that use contextual, naturalinstrument stimuli, and that take advantage of the immediate and random access provided by this system, are described.
\end{abstract}

This article describes the hardware and software components of a tool for psychomusical research based on direct-to-disk quantization of musical-bandwidth signals. Several research paradigms are described that are automated by the system and that take advantage of the immediate and random access to natural musical signals afforded by the system.

\section{Signal Synthesis}

Laboratory systems for digital signal manipulation have been in existence for at least 20 years. Many such systems digitally implement the Fourier equation for additive synthesis of the desired signals. Filtration and timevariant characteristics such as amplitude envelopes are added via arithmetic manipulation of the samples before the result is stored on either tape or disk. Early systems, such as Music V (Mathews, 1969), then proceed with analog-to-digital (A/D) conversion as a separate step, in non-real-time. When utilized for perceptual experiments, the non-real-time constraint results in analog tapes of a small number of orderings of the stimuli. Therefore, the advantage of the digital process is in the precision of signal parameter specification; lost is the ability to randomize stimulus orders for each subject, as well as any increase in signal-to-noise ratio resulting from the digital process.

Hardware implementation of a variety of synthesis techniques has resulted in a plethora of real-time synthesis systems, such as the Synclavier II and the Fairlight. Such synthesizers, when provided with a terminal and the appropriate software, can be programmed by researchers to automate the stimulus ordering process, including the randomization of stimuli for each subject. However, such systems are impossible, or at best difficult, to configure for automated collection of subject responses. Therefore, paper instruments for such collection, or the asynchronous collection of responses using a separate computer, are re-

Exploratory resources for this project, including an Intel System 35 computer, were provided by a grant from Intel Corporation. The author's address is Department of Music, University of California, Los Angeles, CA 90024. quired. In the latter case, the failure of a subject to respond within the allotted time can ruin the data. Responding to the seriousness of this possibility, some researchers abandon the use of complex, time-variant signals, generating static steady-state stimuli using a digital-to-analog (D/A) converter driven in real time by a microcomputer, which is also used to order the stimuli and collect subject responses.

Alternative techniques to Fourier synthesis, such as phase-distortion, pulse-code-modulation, and frequencymodulation synthesis, have been implemented on synthesizers designed for real-time musical performance. The recent implementation of the Musical Instrument Digital Interface (MIDI) protocol permits extensive external control of these synthesizers by a microcomputer, including the specification of signal parameters. However, such hardware configurations do not provide for the manipulation of natural instrument signals, with the exception of sampling keyboards, discussed below. Why should one desire to manipulate natural instrument signals?

\section{Artificial Versus Natural Signals}

Much research in music perception has assumed the validity of results achieved using artificial signals. I have examined this assumption in detail elsewhere (Kendall, 1984, 1986, 1987), and I agree with Plomp (1976, p. 143) that "we have to realize that the study of steady-state tones can only give a limited picture of how sounds in general are preceived [sic]." In an effort to control various dimensions in the vibrational frame of reference, dimensions that are assumed to be a real, sufficient, and necessary description of a physical reality, researchers use steadystate stimuli that bear little resemblance to those normally apprehended in natural musical settings. There is no evidence currently available that allows tacit generalization from such steady-state stimuli to the time-variant stimuli that are so much a part of our musical culture. Use of artificial stimuli assumes that the musical performer has no role in the process of musical communication, or that any role played by a performer is a contaminating or confounding variable. It is just as likely, however, that the 
performer is an important source of information for listeners' perceptual strategies. For example, instrumental timbre cannot be considered outside of the performerinstrument interaction (Kendall, 1986). It is possible that, when we use artificial signals, we find out what subjects can be made to do in experimental settings, not what they do in fact. At the very least, parallel methodologies employing both artificial and natural signals should be employed.

A few studies have employed natural instrument signals, or reconstructed signals based on analyses of natural signals. Luce (1963), Strong and Clark (1967a, 1967b), and Grey (1975) produced early studies of natural instrument signals and timbre perception using large main-frame computers that permitted non-real-time operations, an approach that suffers from the same limitations delineated above for non-real-time synthesis of artificial signals.

\section{Sampling Systems}

Most microcomputer designs for manipulation of natural signals have used random-access memory (RAM) as storage. Data stored in RAM is written to or from hard disk in a separate step. Thus the amount of signal that can be manipulated is severely constrained by available RAM; in addition, since the entire stimulus must be loaded to RAM, access is not immediate. Recently, real musical signals have been captured and manipulated using relatively inexpensive sampling keyboards. However, these devices are also constrained by the limitations of using RAM buffers. Overcoming these limitations involves the use of the hard disk to store incoming and outgoing data; that is, the system must multitask the quantization and disk operations.

One approach to this problem is to have a stand-alone hardware system that samples into its own internal RAM buffer. The microcomputer can poll the buffer's status and transfer data, asynchronous to the sampling, through a port into microcomputer RAM. The microcomputer then writes (or reads) the data onto the hard disk, while the external sampling hardware continues its operations. This approach, similar to that used in the Digisound 16 system, works fairly well, but is not cost effective: The user pays for what amounts to an additional computer in the sampling hardware. Also, depending on the sampling hardware RAM size, disk offload time may exceed the time required to fill and transfer the RAM buffer, resulting in lost data.

A more cost-effective approach is to use $A / D$ and $D / A$ converters interfaced directly to the bus of the microcomputer, the approach taken in the Signal Edit System (Kendall, 1982). The microcomputer CPU is used to transfer data to and from the converters to system RAM on interrupt from a timer and, in the time between samples, to execute hard-disk operations. Unfortunately, the time between samples, a few tens of microseconds, is a serious constraint. For efficiency, the CPU must manipulate the hard disk controller directly; this means the loss of device independence, for the software will function only with a specific disk controller and disk drive. Often only moderate sampling periods can be achieved (approximately $35-40 \mu \mathrm{sec}$ ).

The solution to these problems is the use of direct memory access (DMA). DMA chips permit data transfer between devices and system RAM independently of the normal functioning of the CPU. In addition, more than one DMA device can transfer data simultaneously, as memory accesses are interleaved within the normal bus timing. Thus sampling and disk controller hardware that both utilize DMA can operate simultaneously and asynchronously on the common system memory. The sampleto-disk system described here uses this technique. Such efficiency is achieved that the standard operating system of the microcomputer, which preserves device independence for hard disk and controller, is used. The same microcomputer used for sampling is used for experiment control, data collection, and statistical analysis.

\section{HARDWARE}

The IBM AT architecture provides two Intel 8237A-5 high-performance programmable DMA controllers on the mother board. One is used for 8-bit operations and has two channels available for peripheral accesses. The second is configured for 16-bit transfers, and is used by the hard-disk controller. Data transfer in the disk write and read process is enhanced by the capability of the 80286 microprocessor to copy data to and from memory buffers using the MOVS instruction, which utilizes DMA. Thus the IBM AT architecture is ideally suited for the multitasking required of the sample-to-disk process. In addition, since the DMA controllers are integral to the basic system design, the peripheral hardware component count and design complexity can be kept to a minimum, enhancing cost effectiveness.

The sampling peripheral card is the PC-DMA analog interface board, upgraded for enhanced musical signal performance, designed and marketed by Canetics. ${ }^{1} \mathrm{~A}$ block diagram of the design is provided in Figure 1. The board, available with a maximum quantization accuracy of $\pm 1 / 2$ bit in either 12- or 16-bit formats, provides highspeed (100,000 samples per second) A/D and D/A conversion, and is interfaced with the 8-bit DMA controller on an IBM AT or compatible. The converters can also be software configured for 8-bit operations, conserving storage space at the expense of signal-to-noise ratio (theoretically, signal-to-noise is approximately $6 \mathrm{~dB}$ per bit). Two on-board timers drive the conversion process; it is possible to obtain $A / D$ and $D / A$ conversion simultaneously (in monophonic mode), since a separate DMA channel is available for each converter. A third timer is available to initiate interrupt-driven activities. The multilayer board has separate digital and analog ground planes for reduced noise, and the analog circuitry is powered by an external linear power supply, bypassing the noisy internal power of the microcomputer. A five-pole Butterworth anti-aliasing filter is provided for analog input, and this filter can be inserted into or removed from the signal 


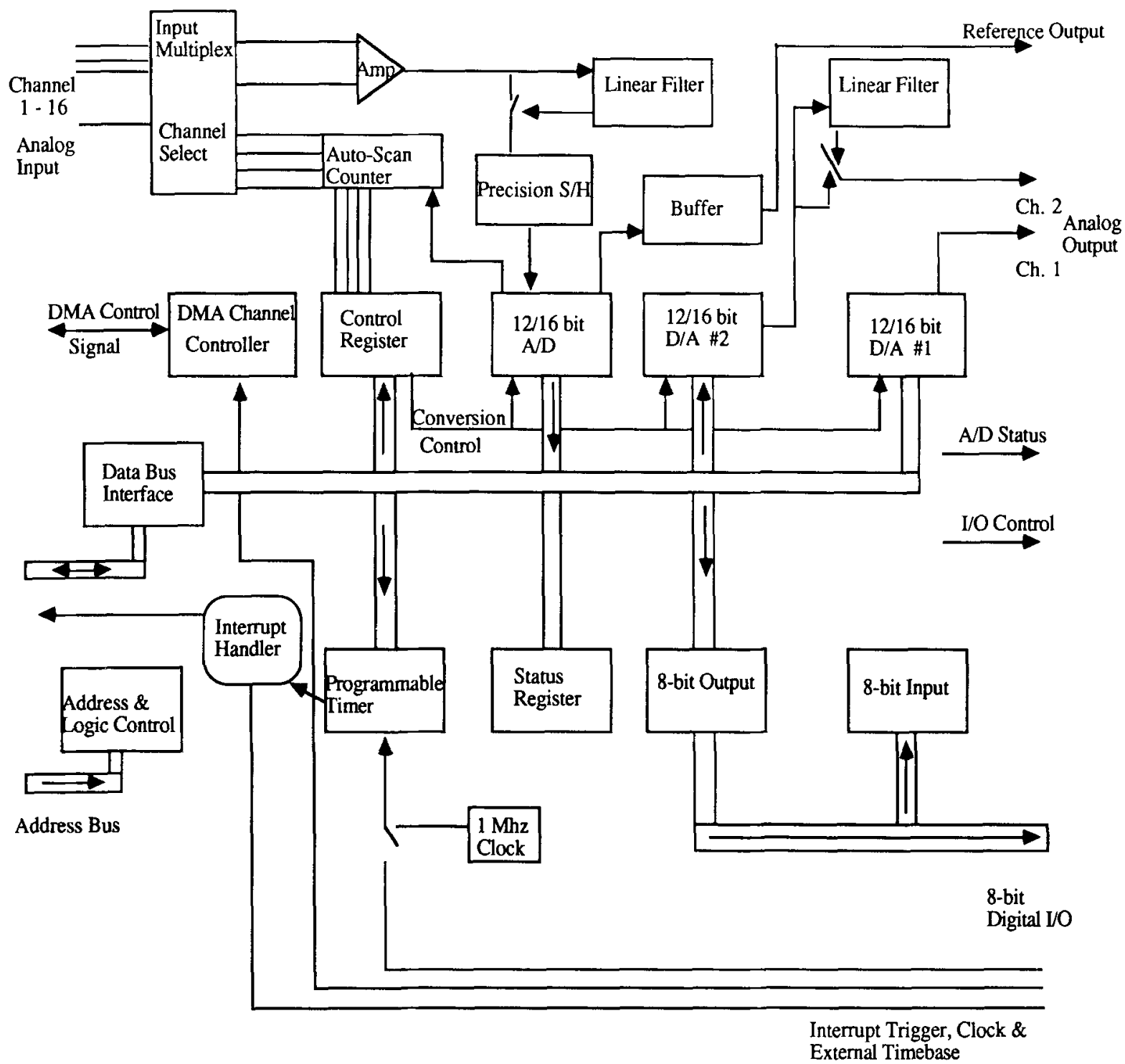

Figure 1. A block diagram of the PC-DMA analog interface board.

path by software commands. A five-pole Butterworth smoothing filter is inserted in the analog output signal path.

A single $\mathrm{A} / \mathrm{D}$ converter and two $\mathrm{D} / \mathrm{A}$ converters are provided. In monophonic mode, the signal from one D/A converter can serve as reference voltage for the other, permitting software control of the output amplitude of the signal in 256 steps. In stereo mode, the analog input to the $\mathrm{A} / \mathrm{D}$ converter is switched between two channels, using an autoscan multiplexer. ${ }^{2}$ For two-channel analog output, both D/A converters are used, with successive samples automatically routed to alternate converters, emulating the $\mathrm{A} / \mathrm{D}$ process.

Finally, the PC-DMA board provides an 8-bit digital input/output port. The sample-to-disk system uses a subject response board consisting of eight momentary contact switches that tie input port lines to ground. The switches are arranged so that their positions on the board are researcher configurable (Figure 2). The subject response board is used in scaling and multiple-choice experimental paradigms, as described below.

The only additional peripheral hardware required is a Microsoft-compatible mouse, which is used by software signal-graphing functions and for subject responses in the categorization paradigm discussed below. Of course, the IBM AT or compatible must include a hard disk of sufficient capacity to store incoming data. Any PC-compatible hard-disk drive and controller can be used. The system as configured at UCLA uses a Seagate ST-4096 drive with a capacity of $81 \mathrm{MB}$. This provides approximately $20 \mathrm{~min}$ of 12-bit monophonic (or 8-bit stereophonic) storage, with a sampling rate of 35,000 samples per second.

\section{SOFTWARE}

The software system consists of two major components: An assembly-language module, which handles the actual sampling process, and a high-level-language module writ- 


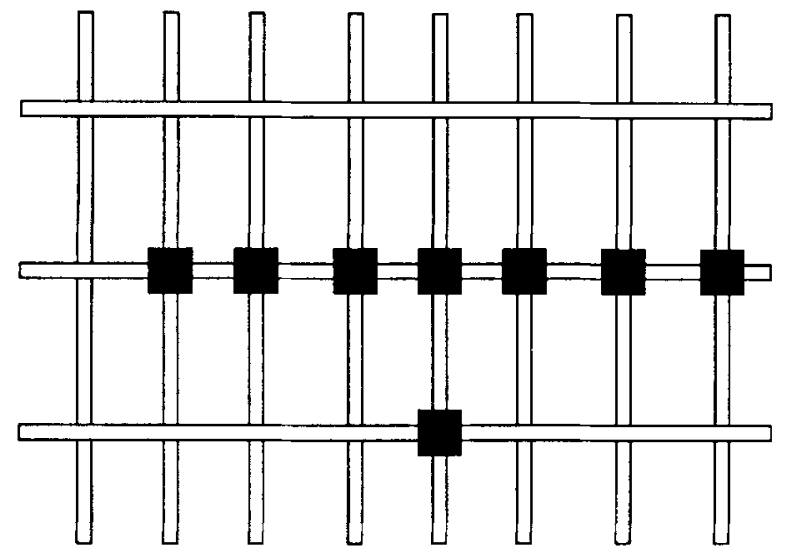

Figure 2. Subject response board switch orientation. Switches can be moved along the tracks and thus configured for different experimental paradigms. The switches close to ground and are read via a digital port on the PC-DMA board.

ten in compiled QuickBASIC (Version 3.0), which provides the user interface.

\section{Assembly-Language Module}

Although the hardware provides nearly automatic sampling to system RAM, the CPU must be used to initiate disk operations. A time-critical aspect of the process is introduced by a limitation of the DMA controller. Intel components used in $80 \mathrm{XXX}$ series architectures address memory in terms of 65,536-byte "pages"; this characteristic is termed "segmented memory addressing," in contrast to the linear memory schemes employed in 68XXX Motorola architectures. The 8237A-5 DMA controller can address memory only within a single page. When the last address is accessed, one of two options must be invoked: (1) The CPU must update the page register to the next RAM page, or (2) the DMA controller must be placed in "autoinitialize" mode, which forces the DMA controller address and count to start over. Note that in the latter case, only a single 65,536-byte page of RAM can be used, since the page register is not automatically incremented. In the former case, any amount of available system RAM can be used; however, the CPU must monitor the DMA and update the page register at the moment the end of the page is reached. Since the CPU is being used for disk operations, if a disk operation is in progress at this moment, data will be lost. The sample-to-disk software uses both procedures for different functions.

For recording ( $\mathrm{A} / \mathrm{D}$ conversion), the assembly module reads a series of sampling and disk parameters passed by the user module, initializing the DMA address and count registers and the PC-DMA timers and multiplexing devices. A/D conversion commences immediately, under the control of the PC-DMA board. The CPU is used to read the DMA count register, which counts down from a page count of 65,534 bytes. When the count reaches 32,767 (a half-page), the CPU invokes the disk operating system disk-write interrupt and commences offload of the top half-page. The PC-DMA A/D process con- tinues, starting over with the top half-page when the bottom half-page is full. When the CPU is finished with writing the top half-page, it checks the count and, if the bottom half-page is full, begins offload of this data. The chase between conversion and disk offload continues until stopped by a keypress on the keyboard. As long as the disk offload of two half-pages requires less time than the A/D conversion of a page, no data is lost. ${ }^{3}$ This critical sample period has been found to be $9 \mu \sec (111,111$ samples per second) for 8-bit data and $19 \mu \mathrm{sec}(52,631.6 \mathrm{sam}$ ples per second) for 12 -bit data. ${ }^{4}$

The D/A playback process for data of more than two pages is the inverse of the above, and stereo sampling is no different than monophonic (except that the sampling rates are per channel for stereo mode). For D/A playback of data of less than two pages, both pages are loaded into RAM, and the CPU is used to monitor and update the DMA page register. This permits looping of signal segments without driving the hard disk controller. The looping of playback is an important component of the user interface.

The machine-code module is assembled into a Microsoft linkable object module. This module is linked to the QuickBASIC module. Sampling is controlled from the high-level language program by a simple function call, with the appropriate parameters set:

CALL DIGITAL(offset,datasize,start.page,start.byte, length.page,length.byte, outmode, function, sample.period, filterstate,gain, source.file\$)

\section{User Module}

The user module is menu driven and is designed for interaction with computer novices. The system has a hierarchically organized set of functions accessed through menu windows (Figure 3). Selection of functions from menus is accomplished by pressing the first letter of the function name.

Because disk operations are handled by the assembly program using calls to Microsoft Disk Operating System (MS-DOS), signal files are standard DOS (disk operating system) files that can be manipulated using any of the tools available in the disk operating system, such as COPY, DIRectory, BACKUP, and SORT. In addition, the user module can invoke DOS services via software interrupts. The user module accesses DOS functions to create a file manipulation environment more intuitive than the cryptic command-line interface provided in DOS.

Data is recorded and played back from files labeled "source files," conventionally located in a special directory called SIGNAL (although any directory can be used). A window at the left of the screen, visible at nearly all times during system operation, provides details such as source file name, length (in samples, pages, and time), quantization accuracy, and mode (mono or stereo). Files are selected via a complete directory system, which locates all signal files on a disk and presents a directory listing; files are selected by highlighting using the arrow keys. 

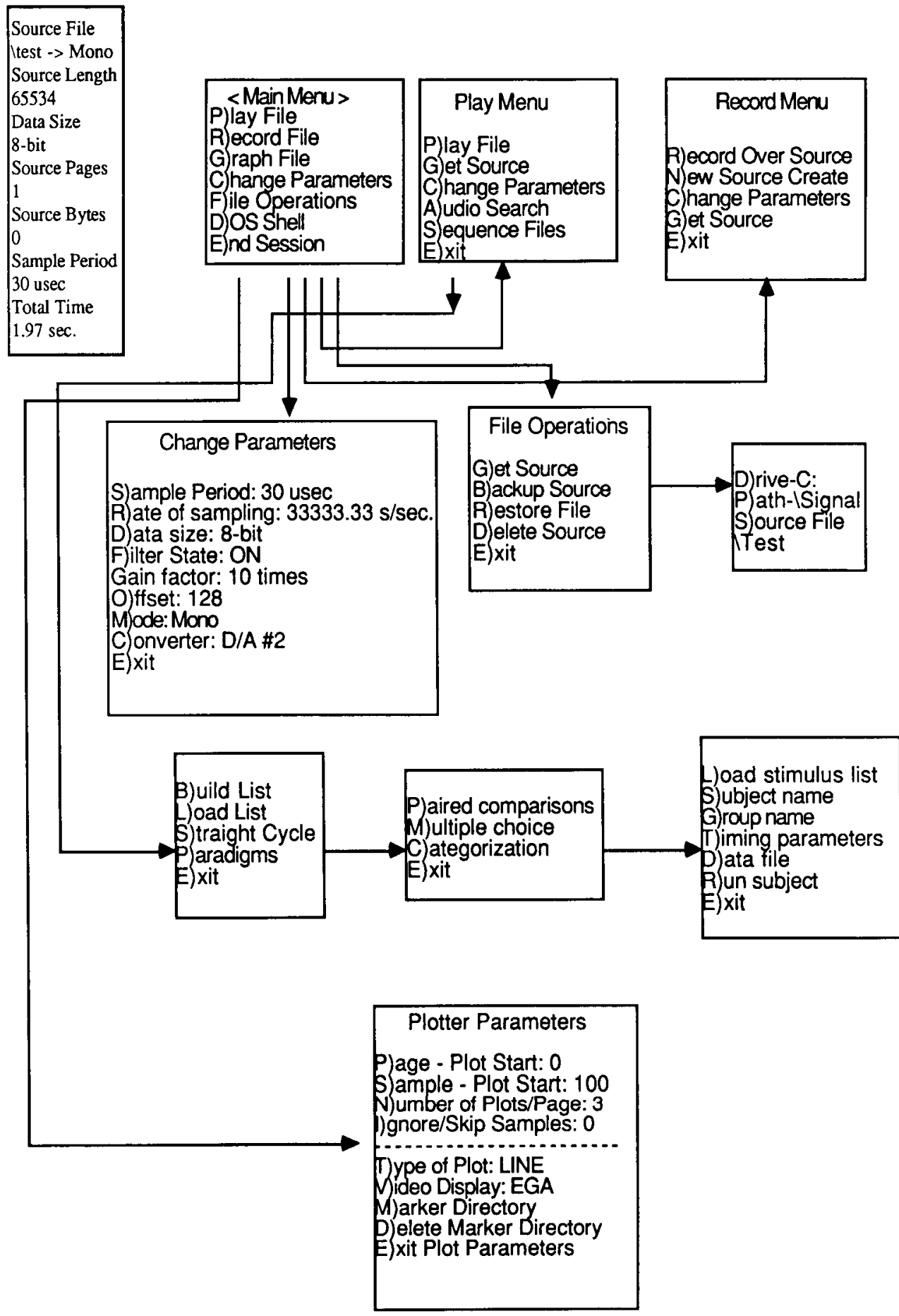

Figure 3. The major menus and functions of the user software module.

New source files are created using the record function, which can optionally overwrite an existing file. Record and playback parameters can be changed from anywhere within the system using the Change Parameters menu, which also serves to provide specific information on the current source file. The entire record/play process consists of simply highlighting or typing in the name of a source file, selecting the desired parameters (sampling rate, quantization size, filter state, and amplifier gain), and invoking the record or play menu function. The recording or playing back of entire files can be interrupted by the operator's pressing any key on the keyboard. At the end of a record function, a data file is created containing the date of creation and all sampling parameter 
information; this data is retrieved automatically on any subsequent selection of the source file. In addition, research notes may be added.

Of course, the basic playback process is of little use in research, other than for verifying that a file has been adequately recorded. To answer research questions concerning performance interpretation or instrumental timbre categorization, it is often necessary to extract from larger files specific phrases or even single notes, and to sequence these for playback in various experimental paradigms. Subsystems for these tasks are provided that automate this process.

Audio search. The Audio Search subsystem permits the opening of an "audio window" into a source file. The signal within the window is played and looped. Using the arrow keys on the keyboard, the operator can open and close this window to any size from a single sample to the entire length of the file. In addition, the window at a specified width can be slid across the signal file. This provides a means of aurally locating signal events of interest, such as note or phrase transitions. Signal segments thus located can be stored in a "marker list," which also stores brief research notes concerning the signal segments. This list and the notes can be displayed at any time, an entry highlighted, and the audio window immediately moved to the marked position. Furthermore, entries in the list can themselves be marked in any order. A function then cycles through the entries in the order selected, permitting aural review of the list. The operator can change any of the sampling parameters at any time, switching from mono to stereo mode or speeding up or slowing down the playback. These capabilities are especially useful for musicological and ethnomusicological transcription, and are used in performance studies in combination with a pitchtracking device, discussed below.

Graphing files. The marker list is transferred to and from other functions of the system. The Graph File subsystem receives the audio marker list. The user can then obtain a signal graph of the segment, and/or a Fourier analysis of the segment (Figure 4). Hamming-window Fourier analysis is provided and displayed in the traditional three-dimensional graph. The Graph File functions also permit scrolling through the signal file and the specification of additional segments obtained through visual analysis; one can also audition these segments. The mouse is used to point to the signal graph display, and a readout of the exact amplitude, time, and sample number at the mouse pointer is provided.

Editing functions are provided that permit marker list segments to be specified and copied, deleted, and/or merged into a destination file. The destination file can thus contain signals with the transients removed, or note or phrase events rearranged in new orders. The destination files become source files upon completion of the editing process.

The final step before experimental paradigm execution is the specification of a larger list containing all signal events to be used in the experiment. The Build List func-

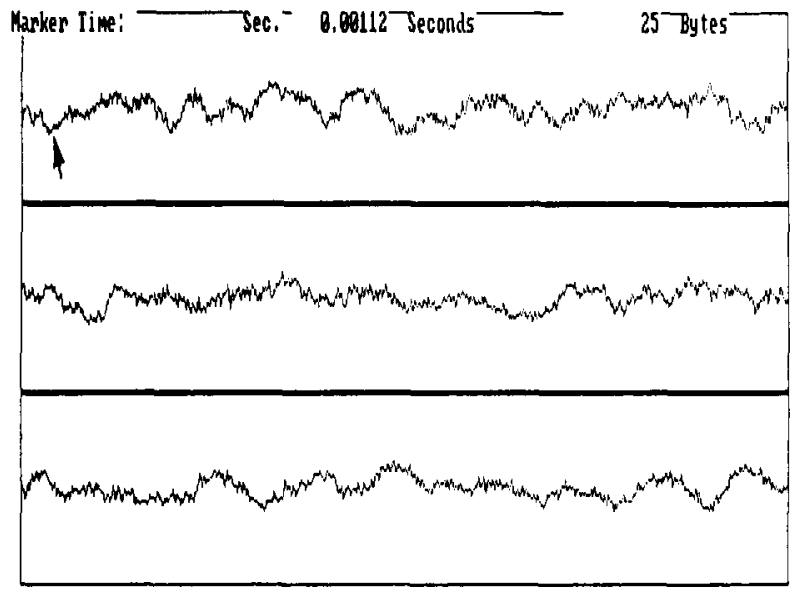

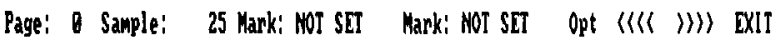

Figure 4. A screen dump of the Graph Files function in operation. Note that the system displays the sample and time position of the mouse pointer. The operator can specify the number of plots on a screen and scroll the display throughout the file.

tion accomplishes this in a very straightforward manner: (1) A list of all signal files is displayed. A highlight is moved across this list. (2) Pressing $<$ ENTER $>$ brings up any segment marker list associated with the larger file. The entire file can also be selected. (3) The user then marks the segments to be included in the longer list. This process is repeated until all segments and/or files are selected. A review function permits additional editing of the longer list, which is then saved for the experimental paradigm function. Files and segments may have any sampling parameters-one can mix different sampling rates, mono versus stereo output, and quantization sizes-since these are coded and stored in the composite list.

Generation of experimental paradigms. At present, three experimental paradigms are automated by the system, the last of which is discussed under a separate heading. In the paired-comparison paradigm, a single composite list is read. The user specifies the number of complete sets to be output, the interstimulus interval, and the response time interval (or an indefinite interval). The system generates a random ordering of the stimuli, records the subject responses entered via the digital port switchbox (described above; see Figure 2), and assembles a similarity/dissimilarity matrix in unscrambled form. This matrix is saved as an ASCII file for direct import into a statistical program. Response latencies may also be optionally recorded. If the subject fails to respond, either no data or a representation of the stimulus pair at a random later time may be recorded.

The multiple-choice paradigm permits two lists to be output such that for every element of one list, the random ordering of all elements of the second list follows. The subject selects which element of the second list is most similar to the model. The automatic subject-response collection operates in the same manner as for the pairedcomparison procedure. 


\section{Research Applications}

Timbre matching. The editing capabilities facilitated through digitization of signals were used to investigate the role of acoustic signal partitions in listener categorization of musical instruments (Kendall, 1986). ${ }^{5}$ Previous investigations of signal transients used only single notes, which always had amplitude-definable attack transients. I was curious about the potency of transients embedded in natural musical phrases. The use of aural and graphical analyses permitted transient definition within phrases; transients between notes were not always definable through the use of an amplitude criterion. Transients were removed from the entire phrases using digital editing processes, a procedure impossible to duplicate using tape-splicing or gating techniques. A series of signals without transients, natural signals, and signals with static steady-states derived from single periods of the natural signal were automatically sequenced in the multiple-choice paradigm outlined above. In contrast to the findings of over 15 previous studies using single-note contexts, I found that transients were not criterial in defining the characteristic sounds of instruments when the stimuli were musical phrases; musical context provided information for listener categorization. The time-variant steady-state was a much more important signal partition for such instrument identification than were the attack and legato transients.

Categorization. Current research with the present system is directed toward investigating listener strategies for the categorization of musical structures. The categorization paradigm used in these investigations, and implemented in the present hardware and software environment, would be impossible without immediate random access to a large number of relatively long (approximately 1 min) musical excerpts.

The procedure is as follows: $A$ theme and a set of 32 melodic variations (all in the same key, but differing along many other musical dimensions), performed by a professional pianist and by a computer-controlled piano sampling keyboard, are recorded into the system. The recording is made under simulated concert conditions, but without an audience: The pianist plays the variations in an auditorium as a complete work without stopping. The intent of this procedure is to preserve performer information to be found in the transitional relations of one variation to the next. The audio search functions are used to segment the performances into variations, which are cataloged in a marker list. This list is used for experimental paradigm execution.

The marker list is randomly scrambled for each subject. The bottom of a computer screen is used to display a series of homogeneously colored rectangles, each of which represents one of the melodic variations. This area is the "ungrouped" portion of the screen; the area above this is used to establish stimulus groups. The mouse and pointer are used to point to the rectangles. Clicking the left mouse button on a rectangle begins playback of the associated variation.
The subject's task is to group the examples into homogeneous clusters (Figure 5). When the subject points to an ungrouped rectangle and clicks the right mouse button, the associated stimulus becomes the beginning of a new group. When the subject points to an ungrouped rectangle, clicks the left button, then points to a grouped rectangle and clicks the left and right buttons together, the ungrouped rectangle is moved into the selected group. Similarly, rectangles may be moved from group to group, and by pointing to a grouping bar at the top of a group column, the subjects can merge entire groups. Thus the subject can review grouping decisions and make changes at any time. The computer records the stimulus groupings as well as time spent listening to, and the number of grouping changes made with, specific stimuli.

I am interested in whether this categorization paradigm produces results comparable to those obtained with multidimensional scaling of similarities and dissimilarities. Therefore, the same marker list used for the categorization paradigm is loaded for use by the paired-comparisons paradigm. The results, a data triangle of similarities, are analyzed through multidimensional scaling; the dimensional solutions should confirm or disconfirm the results of the categorization paradigm.

Results from this investigation will indicate whether there are well- or ill-defined categories for these musical excerpts, and whether a criterial attribute model or

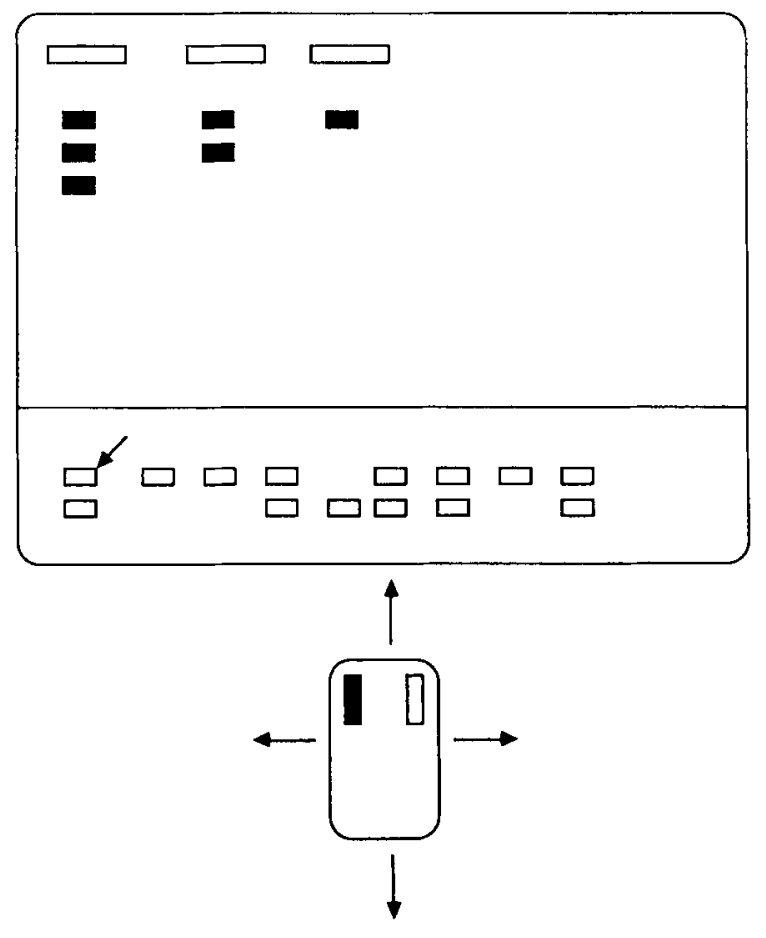

Figure 5. Screen display of the categorization paradigm. Dark rectangles have been clustered under grouping bars at the top of the screen. The white rectangles are stimuli that have yet to be grouped. The figure shows the left mouse button depressed, resulting in the playback of the signal associated with the selected rectangle. 


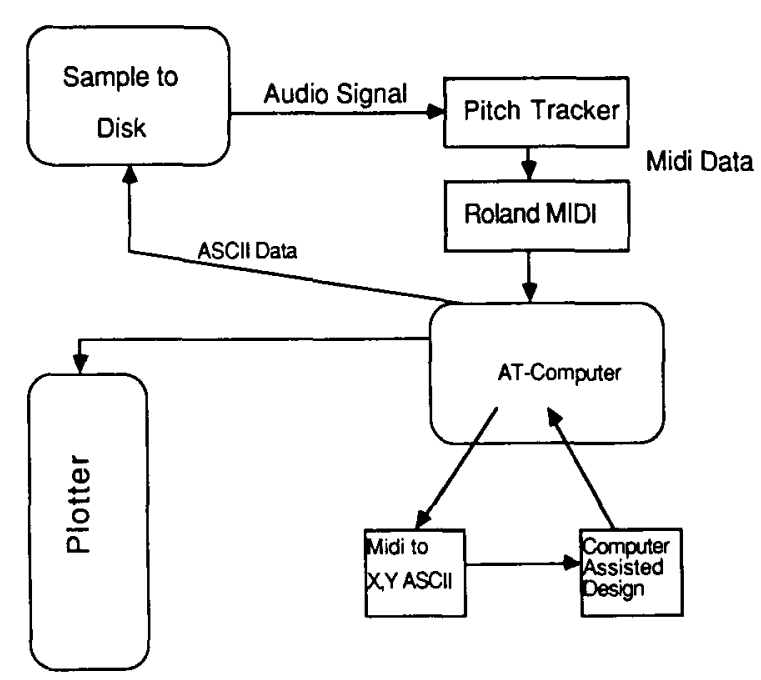

Figure 6. A block diagram of the two-computer arrangement used for monophonic natural-instrument performance analysis.

probabilistic model best fits the subject behaviors. Furthermore, the pianist and computer renditions can be compared to determine whether stylistic information is provided by the performer, whether such information is used in categorization decisions, and along what dimensions such performer information is important.

Performance analysis. To aid in the analysis of nonkeyboard monophonic performances, I have attached the sample-to-disk system to another IBM computer system, a Fairlight Pitch-Tracker, a Roland MPU-401 MIDI, and a graphic plotter (Figure 6). Instrumental phrases are sequenced from the sample-to-disk system; the monophonic analog signal is sent to the Pitch-Tracker. The PitchTracker uses amplitude and periodicity analysis to produce pitch frequency data in terms of diatonic scale degrees and cents (100th semitone) microtonal data. Since the Pitch-Tracker cannot display more than two or three screens of data, musical phrases longer than a few seconds cannot be analyzed. However, the pitch data is retrievable in MIDI format through connections on the PitchTracker. The second computer system is programmed to receive the MIDI data and translate it into time/pitch (horizontal/vertical) coordinates. These coordinates are converted into ASCII notation, and a file is written that contains commands which can be read by a computerassisted design (CAD) program. Therefore, the time/pitch data is plotted in the CAD system, which is normally set so that 12 " equals a quarter-beat along the time dimension and 12" equals an octave along the frequency dimension. As the CAD program provides functions for zooming images, measuring distances, copying sections, and the like, it is an easy task to create plots for comparative analysis of signals. These plots are sent to a plotter for hard copy.

\section{Future Directions}

Canetics is completing a digital signal coprocessing board that operates at exceptionally high speeds and is fully compatible with the PC-DMA board. Thus, it will be possible to perform real-time Fourier transforms on the signal as the signal is being written to or read from the hard disk. Current plans call for the integration of this high-speed device into the current software, thereby providing the capability of having a periodicity tracking filter suitable for timbre experiments.

\section{REFERENCES}

Canetics, Inc. (1986). PC-DMA User Manual. Pasadena, CA: Author. GREY, J. (1975). An exploration of musical timbre. (Doctoral dissertation. published as Department of Music Report STAN-M-2). Stanford, CA: Stanford University, Center for Research in Computer Applications in Music and Acoustics.

KENDALL, R. (1982). Documentary report on the signal edit system (Internal report). Storrs: University of Connecticut, Department of Music, Music Performance Analysis Laboratory.

KendaLL, R. (1984). The role of transients in listener categorization of musical instruments: An investigation using digitally recorded and edited musical phrases. Dissertation Abstracts International, 45(8), 2297A. (University Microfilms No. 82-25754)

KeNDALL, R. (1986). The role of acoustic signal partitions in listener categorization of musical phrases. Music Perception, 4, 185-214.

KENDALL, R. (1987). Model-building in music cognition and artificial intelligence. In Proceedings of the Artificial Intelligence and Advanced Computing Technology Conference/East (pp. 183-196). Chicago: Tower Conference Management.

LUCE, D. (1963). Physical correlates of nonpercussive musical instrument tones. Unpublished doctoral dissertation, Massachusetts Institute of Technology, Cambridge.

MATHEWs, M. V. (1969). The technology of computer music. Cambridge, MA: MIT Press.

Plomp, R. (1976). Aspects of tone sensation. London: Academic Press. STrong, W., \& Clark, M. (1967a). Perturbations of synthetic wind instrument tones. Joumal of the Acoustical Society of America, 41, 277-285. Strong, W., Clark, M. (1967b). Synthesis of wind-instrument tones. Journal of the Acoustical Society of America, 41, 39-52.

\section{NOTES}

1. Canetics, Inc., may be contacted at P. O. Box 70549, Pasadena, CA 91107.

2. For applications that require stereo sampling where this phase shift might be undesirable, it is possible to compensate using the transfer function $H(w)=(A(w) / B(w)) * \exp (-j T w)$, where $a(w)$ and $b(w)$ are Fourier transforms of the two channel inputs and $T$ is the intersample interval (Canetics, 1986). For a sampling rate of 41,000 samples per second, the phase shift of a $440-\mathrm{Hz}$ sine would be approximately equal to $.25^{\circ}$.

3. This description oversimplifies the actual logic, which is predicated on the fact that some disk operations require more mechanical drive motion and software housekeeping than others. As a result, some half-pages take longer to offload than others. This observation also illustrates why the autoinitialize-mode-DMA on a single page is preferable to CPU intervention for page update.

4. An explanation of the specific variables that influence this rate is beyond the scope of this paper. Clock speed is the least significant factor, but BIOS efficiency and hard disk controller speed are important. The values given are for a $10-\mathrm{MHz}$ AT clone using generic, Taiwanbuilt hardware and Phoenix BIOS.

5. This investigation was carried out on the early Northstar microcomputer implementation of what has become the present sample-to-disk system (see Kendall, 1984). 